\section{To be red or white: lineage commitment and maintenance of the hematopoietic system by the "inner myeloid"}

\author{
Hiroki Kato ${ }^{1,2,3}$ and Kazuhiko Igarashi ${ }^{1}$ \\ ${ }^{1}$ Department of Biochemistry, Tohoku University Graduate School of Medicine, Sendai, \\ Japan; ${ }^{2}$ Department of Hematology and Rheumatology, Tohoku University Graduate \\ School of Medicine, Sendai, Japan and ${ }^{3}$ Present address, Center for Regenerative \\ Medicine, Massachusetts General Hospital, Boston, MA, USA
}

\section{ABSTRACT}

$\square$ ifferentiation of hematopoietic stem and progenitor cells is tightly regulated depending on environmental changes in order to maintain homeostasis. Transcription factors direct the development of hematopoietic cells, such as GATA-1 for erythropoiesis and PU.1 for myelopoiesis. However, recent findings obtained from single-cell analyses raise the question of whether these transcription factors are "initiators" or just "executors" of differentiation, leaving the initiation of hematopoietic stem and progenitor cell differentiation (i.e. lineage commitment) unclear. While a stochastic process is likely involved in commitment, it cannot fully explain the homeostasis of hematopoiesis nor "on-demand" hematopoiesis in response to environmental changes. Transcription factors BACH1 and $\mathrm{BACH} 2$ may regulate both commitment and on-demand hematopoiesis because they control erythroid-myeloid and lymphoid-myeloid differentiation by repressing the myeloid program, and their activities are repressed in response to infectious and inflammatory conditions. We summarize possible mechanisms of lineage commitment of hematopoietic stem and progenitor cells suggested by recent findings and discuss the erythroid and lymphoid commitment of hematopoietic stem and progenitor cells, focusing on the gene regulatory network composed of genes encoding key transcription factors. Surprising similarity exists between commitment to erythroid and lymphoid lineages, including repression of the myeloid program by $\mathrm{BACH}$ factors. The suggested gene regulatory network of $\mathrm{BACH}$ factors sheds light on the myeloid-based model of hematopoiesis. This model will help to understand the tuning of hematopoiesis in higher eukaryotes in the steady-state condition as well as in emergency conditions, the evolutional history of the system, aging and hematopoietic disorders.

\section{Introduction}

Hematopoietic stem cells (HSC) possess the abilities of self-renewal and multilineage differentiation, including that to red and white blood cells and platelets (i.e., erythrocytes, megakaryocytes, innate immune cells and acquired immune cells). ${ }^{1}$ Salient aspects of the hematopoietic system include its potential to produce huge numbers of cells with distinct functions throughout the life span of a human and its tunability, by which the output is balanced in response to environmental changes, such as from the steady state to an infectious state.

Erythrocytes are the most abundant cells in the human body, accounting for around $70 \%$ of the total cell number ${ }^{2}$ and $200 \times 10^{9}$ erythrocytes are produced daily. ${ }^{3}$ Although the estimated number of white blood cells is much lower than that of erythrocytes, ${ }^{2}$ the short life span of myeloid cells necessitates the production of a huge number of these cells as well. For instance, the circulating half-life of neutrophils is $6-8 \mathrm{~h}$, and their estimated production rate is $50-100 \times 10^{9}$ cells per day. ${ }^{4}$ In line with this, label tracing analyses of HSC have revealed that the production rate of erythroid-myeloid progenitors is about 180 times higher than that of lymphoid progenitors in unperturbed hematopoiesis. ${ }^{5}$ Thus, hematopoietic

Ferrata Storti Foundation

Haematologica 2019

Volume 104(10):1919-1927

\section{Correspondence:}

KAZUHIKO IGARASHI

igarashi@med.tohoku.ac.jp

Received: February 14, 2019.

Accepted: May 10, 2019.

Pre-published: September 12, 2019.

doi:10.3324/haematol.2019.216861

Check the online version for the most updated information on this article, online supplements, and information on authorship \& disclosures: www.haematologica.org/content/104/10/1919

(C)2019 Ferrata Storti Foundation

Material published in Haematologica is covered by copyright. All rights are reserved to the Ferrata Storti Foundation. Use of published material is allowed under the following terms and conditions:

https://creativecommons.org/licenses/by-nc/4.0/legalcode. Copies of published material are allowed for personal or internal use. Sharing published material for non-commercial purposes is subject to the following conditions: https://creativecommons.org/licenses/by-nc/4.0/legalcode, sect. 3. Reproducing and sharing published material for commercial purposes is not allowed without permission in writing from the publisher. 
stem and progenitor cells (HSPC) have an exceptionally vigorous ability to produce huge numbers of cells constitutively. To maintain its homeostasis, the production pace of each mature cell lineage must be tightly regulated according to environmental changes ("on-demand" hematopoiesis).

Infection is one of the most common challenges facing hematopoiesis and evokes the induction of myelopoiesis as well as the suppression of erythropoiesis. ${ }^{6}$ Induced myelopoiesis during an infection is an effective way of eliminating pathogens, whereas the repression of erythropoiesis may help by limiting the availability of nutritional iron supply to pathogens and/or red blood cells as a target of infection, such as in malaria infection. ${ }^{7}$ However, infection and prolonged inflammation can cause anemia of inflammation, which is the second-most prevalent type of anemia after iron-deficiency anemia. ${ }^{8}$

As with infection, the activity of HSPC is also altered with aging and in various disease conditions. The production of erythrocytes is often reduced in elderly people, leading to anemia, ${ }^{9}$ and acquired immunity becomes less effective with aging, which can result in increased susceptibility to infectious diseases and malignancy in the elderly. ${ }^{10,11}$ In contrast, the production of myeloid cells often increases with aging. ${ }^{11,12}$ This skewed trajectory selection of HSPC induced by aging might be related to the development of aging-related hematopoietic disorders, such as myelodysplastic syndrome (MDS). Although the molecular mechanisms by which the function and differentiation of HSPC are altered by aging are still largely unknown, emerging evidence suggests contributions of inflammation and/or inflammatory signaling to aging of HSPC. ${ }^{13}$

In order to facilitate the treatment of infection-associated and aging-associated diseases, it is important to understand the mechanisms by which the differentiation trajectory of HSPC and their commitment are defined at steady state and how these mechanisms are altered in inflammatory conditions. Although accumulating knowledge has shown that transcription factors (TF) play central roles in the differentiation of HSPC, the precise mechanisms underlying the initial lineage commitment and "on-demand" hematopoiesis are still unclear and cannot be wholly attributed to TF. Complicating matters further is the fact that HSPC are substantially heterogeneous and many appear to be already committed to certain differentiation fates. ${ }^{14-16}$ It is, therefore, important to distinguish the roles of TF in initiating the commitment of uncommitted progenitors from that of their executive roles in the progression of differentiation toward a particular fate. Thus, the actual point of differentiation commitment may need to be reconsidered.

We recently demonstrated the roles of $\mathrm{BTB}$ and $\mathrm{CNC}$ homology (BACH) TF, BACH1 and BACH2 (BACH factors), in instructing erythroid-myeloid progenitors and lymphoid-myeloid progenitors to respond to environmental changes. ${ }^{17-19}$ BACH factors form heterodimers with small Maf proteins to bind to the Maf recognition element (MARE), which contains an AP-1 site. ${ }^{20}$ Importantly, AP-1 sites play central roles in hematopoietic cell immune reactions. ${ }^{20,21}$ BACH1 plays important roles in the maturation of erythrocytes by balancing heme and globin proportions, especially in the condition of iron deficiency, ${ }^{22}$ whereas BACH2 plays important roles in the development of plasma cells, memory B cells, regulatory $\mathrm{T}$ cells and memory $\mathrm{T}$ cells. ${ }^{23-30}$ These findings suggest ubiquitous roles for $\mathrm{BACH}$ factors in the maintenance of homeostasis in both steady-state and inflammatory-state hematopoiesis, as described below.

In this review, we summarize the latest findings concerning the mechanisms underlying lineage commitment of HSPC and potential questions to be addressed. We also discuss gene regulatory networks composed of genes encoding key TF which compete for lineage identities and downstream genes encoding effector molecules, focusing particularly on erythroid-myeloid and lymphoid-myeloid differentiation, two major points of commitment in HSPC differentiation. In addition, we review the roles of $\mathrm{BACH}$ factors in the myeloid-based model of hematopoiesis, which may provide a new concept of the fundamental mechanism in HSPC differentiation, and its meaning in an evolutionary perspective. We also discuss the diverse functions of $\mathrm{BACH}$ factors in mature hematopoietic cells as a strategy to cope with environmental changes through the maintenance of hematopoiesis. Finally, we describe how changes in lineage commitment can lead to diseases, such as anemia of inflammation and MDS.

\section{Lineage commitment of hematopoietic stem and progenitor cells}

The multipotency of HSC has been demonstrated by single-cell transplantation into irradiated mice. ${ }^{31}$ This led to vigorous investigations into how HSC differentiate into diverse lineages of cells with distinct functions. The isolation and characterization of progenitor cells led to the idea that HSC gradually and systematically lose multipotency, generating progenitor cells with limited differentiation trajectories, such as common myeloid progenitors (CMP), ${ }^{32}$ which can generate myeloid cells and erythroid cells but not lymphoid cells. On the other hand, although all blood cells derive from a $\mathrm{FLT}^{+}$multipotent progenitor stage, ${ }^{33}$ lymphoid-primed multipotent progenitors (LMPP) preferentially differentiate into lymphoid cells and myeloid cells with a low differentiation potential to erythroid cells. ${ }^{34-36}$ This led to the recognition that HSC eventually lose their ability to differentiate to erythroid or lymphoid cells, leaving erythroid-myeloid bifurcation and lymphoid-myeloid bifurcation as the two major subsequent points of branching.

Such subpopulations of progenitors have been defined based on the presence or absence of a limited number of cell surface markers, leaving the potential impurity of these subpopulations as a limitation. Indeed, recent comprehensive, single-cell transcriptomic analyses have shown that the known subpopulations of HSPC are composed of heterogeneous cells in terms of gene expression. ${ }^{14,16}$ In addition, in vitro and in vivo single-cell differentiation analyses have shown that only a limited number of cells in progenitor cell populations can produce multilineage mature cells and that a majority of the cells in these populations are already committed to become unilineage mature cells. ${ }^{14,15}$ Furthermore, an in vivo HSC chasing system using endogenous fluorescent tagging revealed that the differentiation trajectory of HSC is already oriented to specific lineage outputs by epigenetic memory. ${ }^{37}$ These observations raise two possibilities: (i) HSPC can be further divided into subpopula- 
tions representing pure differentiation bifurcation points; or (ii) lineage commitments occur only in HSC, whereas each progenitor population is a mixture of committed cells sharing the same cell surface markers at the time of isolation. Since single-cell differentiation analysis from CMP and LMPP showed that a minor part of these populations can produce multilineage mature cells, ${ }^{14,15}$ there might be further subpopulations that represent actual bifurcation points of erythroid-myeloid or lymphoidmyeloid differentiation. However, whether or not these hypothetical subpopulations can be defined using additional cell surface markers remains unclear. Before addressing these two possibilities, we need to stop and consider the potential limitations of recent studies using single-cell analyses. A single-cell transcriptomic analysis is a 'snapshot' observation. Therefore, if a set of genes shows dynamic fluctuations in expression with coherent patterns in cells of a specific subpopulation, these cells might be considered heterogeneous. However, these subpopulations can be homogenous when time-dependent fluctuations are considered, like those observed in neural progenitors. ${ }^{38}$ In addition, despite the importance of the microenvironment for HSPC biology, ${ }^{39}$ an ex vivo single-cell transcriptomic analysis is devoid of anatomical information. Furthermore, single-cell in vivo or in vitro differentiation analyses can only examine the differentiation potential under stress and/or artificial conditions (i.e., cell sorting, culture and transplantation into irradiated mice), which can skew the original differentiation trajectory of progenitor cells, ${ }^{40}$ possibly by altering activities of critical TF whose expression is thought to be maintained to some extent for multilineage priming, ${ }^{41}$ a state in which multiple, conflicting lineage-affiliated genes can be induced or co-expressed. In other words, there is a chance that progenitor cells with unilineage output potential in perturbed conditions still possess multilineage output potential in an unperturbed condition. Recent studies using single-cell analyses may, therefore, lack information about the dynamics (time and threedimensional information) of lineage commitment, ${ }^{42}$ especially regarding unperturbed hematopoiesis. Potential effects of circadian rhythm in HSC differentiation might also have to be considered. ${ }^{43}$ The analysis of entropy in gene expression within single cells ${ }^{44}$ and the threedimensional detection of transcriptomics ${ }^{45}$ might be helpful. Remarkably, recent in vivo barcoding analyses give new support to the existence of a hierarchical development model in hematopoiesis. ${ }^{46,47}$

We must therefore reconsider the actual point at which lineage commitment occurs. An alternative approach to define such a point involves using the regulatory mechanisms of the differentiation of HSPC. To this end, the precise understanding of gene regulatory networks governed by TF may provide a dynamic view of lineage commitment.

This leads us to the second point that should be considered: how are the differentiation trajectories shaped and restricted along the path of differentiation? Several models of lineage commitment have been proposed, showing that TF are critical to shaping and resolving the patterns of lineage-affiliated gene expression. ${ }^{48-50}$ One model features a network of two TF, each promoting differentiation into a specific lineage. If the expression of these two TF is inhibited in a mutual manner and thus they induce their own expression, they can define two cell types with distinct expression patterns of the two TF and thus their downstream target genes (Figure 1). ${ }^{48}$ Machine-learning methods using single-cell transcriptomic data support the notion that gradual, stochastic changes in a few TF have a strong influence on the lineage commitment of progenitor cells. ${ }^{50}$ Such a gene regulatory network may therefore dictate lineage commitment.

However, it has been unclear how one or the other of these TF are initially upregulated or downregulated upon lineage commitment. Stochastic fluctuation in these TF may be involved, ${ }^{48}$ but the output of hematopoiesis should be dynamically tuned in response to diverse stressors, as HSPC produce huge numbers of mature cells daily in a fine balance, as noted above. This property of the hematopoietic system may not therefore be fully explained merely by the stochastic fluctuation of TF. The differentiation trajectory of HSPC must be tightly controlled by responding to environmental changes in order to maintain homeostasis. This means that environmental factors, including pathogen-associated molecular patterns (PAMP) and damage-associated molecular patterns (DAMP), ${ }^{51}$ may affect the cell-intrinsic TF of gene regulatory networks that control the differentiation trajectory. It is therefore important to understand how cell-intrinsic systems of TF are connected to extrinsic signals.

\section{The gene regulatory network for erythroid lineage commitment}

Erythroid cells are derived from progenitor cells that possess the ability to differentiate into erythroid or myeloid cells. ${ }^{1,52} \mathrm{CMP}$ have long been considered to represent a bifurcation point of erythroid-myeloid differentiation. ${ }^{32}$ However, single-cell analyses have challenged this notion. A single-cell RNA sequencing analysis of ckit ${ }^{+}$Sca1-lineage bone marrow cells revealed at least seven different subpopulations with lineage priming at the transcriptomic level. ${ }^{16}$ Importantly, no subpopulations with multilineage priming were observed in that

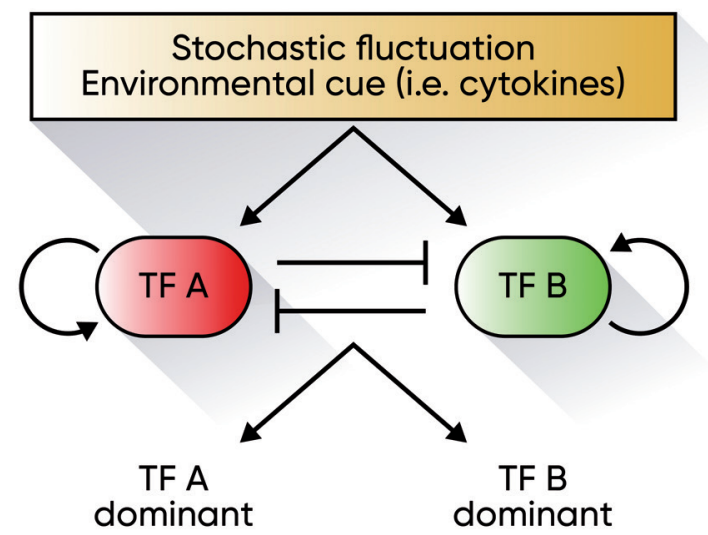

Figure 1. Stochastic model of lineage commitment. Transcription factors (TF) $A$ and TF B play important roles in determining cell differentiation. If these two TF activate themselves and work in a mutually exclusive manner, slight stochastic fluctuations that alter the ratio of TF A to B can affect cell fate. 
study. In addition, a barcoded progenitor cell transplantation analysis revealed that the majority of CMP can differentiate into only erythroid or myeloid cells after transplantation. ${ }^{15}$ Therefore, CMP are a highly heterogeneous population of progenitor cells, and the dominant populations in CMP are already committed to erythroid or myeloid differentiation. However, it should be noted that these findings were obtained from a "snapshot" analysis, which may have overlooked the plasticity of differentiation potential or gene expression patterns in CMP. Indeed, the introduction of specific TF (such as GATA-1 and DDIT3) into myeloid lineage progenitors can switch the lineage output to the erythroid lineage, suggesting the existence of plasticity under the control of $\mathrm{TF}$ in erythroid-myeloid progenitors. ${ }^{53,54}$ This idea is not surprising when we consider the fact that TF often alter the epigenetic modifications for lineage commitment (e.g., via pioneer $\mathrm{TF}^{55}$ ) and that epigenetic changes per se are reversible. ${ }^{56,57}$ Therefore, the observed subpopulations of CMP may show plasticity under physiological conditions, which can be masked during transplantation. In this context, it is still too early to conclude that CMP are heterogeneous populations of already committed progenitors. Further investigations combining single-cell chasing with the comprehensive measurement of epigenomes and transcriptomes in unperturbed conditions will be needed.

In the view of the gene regulatory networks at the erythroid-myeloid bifurcation, key $\mathrm{TF}$, including the CCAAT-enhancer-binding protein (C/EBP) family, ${ }^{58,59}$ PU. $1^{60}$ and GATA-1, ${ }^{61}$ play essential roles in erythroid or myeloid differentiation, which might operate the erythroid-myeloid bifurcation at the level of CMP or multipotent progenitors. Given that GATA-1 and PU.1 show mutually exclusive expression patterns during erythroid and myeloid differentiation and repress each other and activate themselves, the gene regulatory network of GATA1 and SPI1 (encoding PU.1) may determine the bifurcation of myeloid and erythroid cells. ${ }^{48}$ In this model, stochastic alterations in the ratio of GATA1 to PU.1 activity might initiate the differentiation. However, a recent study found that GATA1 and SPI1 are not coexpressed in CMP. ${ }^{62}$ Upon erythroid differentiation, the expression of GATA1 commences with a substantial lag after the cessation of SPI1 expression. In contrast, upon myeloid differentiation, no progenitor cells showed a period with GATA1 expression. ${ }^{62}$ Thus, GATA-1 may not be the initiator of erythroid differentiation but just the executor of the erythroid development from progenitor cells whose erythroid commitment has already been defined by unknown factors. Recent single-cell proteomic analyses additionally revealed that the TF KLF1 and FLI1 play important roles in the bifurcation of erythroid and megakaryocytes. ${ }^{63}$

Since erythroid cells and myeloid cells are rigorously produced from progenitor cells every day, as described above, there should exist a mechanism to fine tune the differentiation trajectory shift of erythroid-myeloid common progenitor cells depending on the demand, which can vary with environmental changes. For instance, infections and inflammation induce myeloid differentiation and reduce erythroid differentiation, which can lead to anemia of inflammation. It has long been accepted that the major cause of this form of anemia is a disorder of iron utility for erythroid maturation caused by the induction of hepcidin, which inhibits iron uptake and recycling. ${ }^{8}$ However, since iron supplementation for the treatment of anemia of inflammation is still controver$\mathrm{sia}^{8,64}$ and infections as well as inflammation can induce a shift in the differentiation trajectory at the level of erythroid-myeloid progenitors ${ }^{65}$ there may be other factors that modulate the differentiation trajectory of progenitors, depending on environmental changes.

We recently reported that $\mathrm{BACH}$ factors are required for the efficient commitment of HSPC to an erythroid fate. ${ }^{19} \mathrm{BACH}$ factors inhibit the expression of $\mathrm{Cebpb}$, the gene encoding the TF C/EBP $\beta$, which plays an indispensable role in emergency myelopoiesis. ${ }^{59}$ Importantly, $\mathrm{BACH}$ factors and C/EBP $\beta$ exert opposite effects on their downstream target genes: $\mathrm{BACH}$ factors repress a set of myeloid-affiliated genes, whereas C/EBP $\beta$ activates these genes at the same genomic loci. Since both BACH factors and the C/EBP family can bind to AP-1 motifs, the balance between repression and activation via the AP-1 motif appears to be critical for determining myeloid fate $^{21,66}$ (Figure 2). Since infectious stimuli repress the expression of $\mathrm{BACH}$ factors and induce $\mathrm{C} / \mathrm{EBP} \beta$ expression, ${ }^{18,19}$ the gene regulatory network of these TF genes can fluctuate in response to environmental input between two states, which correspond to erythroid and myeloid fates.

\section{The gene regulatory network for lymphoid lineage commitment}

Lymphoid cells are also derived from common progenitor cells that possess the ability to differentiate into lymphoid or myeloid cells. LMPP are now considered such common progenitors. ${ }^{1,34,52}$ Similar to the single-cell transcriptomic observations in CMP, a single-cell analysis of LMPP also showed that LMPP are a heterogeneous population. ${ }^{14}$ For instance, a single-cell in vitro differentiation assay showed that most LMPP were only able to differentiate into either myeloid or lymphoid cells. ${ }^{14}$ Therefore, most LMPP may be cells whose differentia-

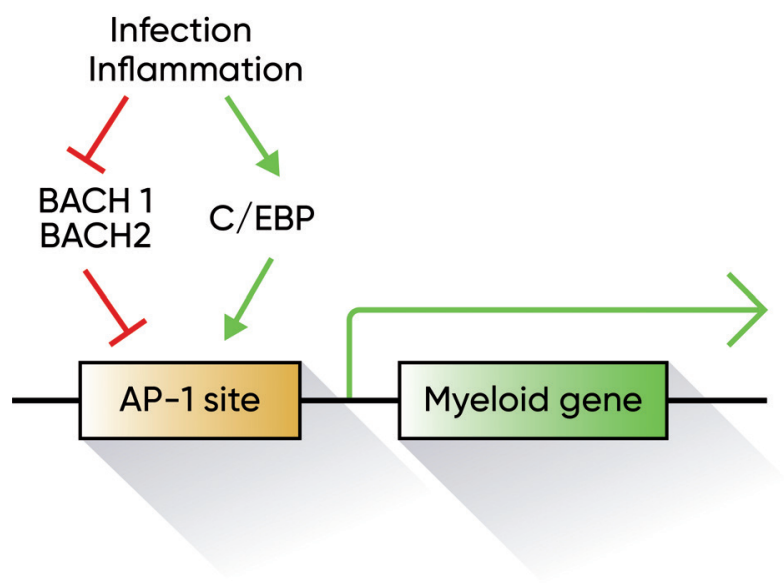

Figure 2. Control of myeloid gene expression by BACH and C/EBP transcription factors in a state of infection. The BACH and C/EBP transcription factors (TF) repress and activate, respectively, myeloid gene expression by binding the same genomic loci. Infection/inflammation-induced alteration of these TF can affect myeloid gene expression, depending on the environment. 
tion commitment has already been decided. However, there may be pitfalls associated with these observations, similar to those regarding erythroid-myeloid bifurcation. The presence of myeloid-lymphoid progenitors is also supported by the findings of an analysis of lymphopoiesis in human embryos. This progenitor population first emerges as a myeloid progenitor and later acquires myeloid-lymphoid bipotential, co-expressing genes affiliated with the two lineages in single cells. ${ }^{67}$

A number of key TF have been identified as important factors for the development of lymphoid cells, including the C/EBP family, PU.1, E2A, IKAROS and FOXO1. An analysis combining RNA sequencing and chromatin immunoprecipitation sequencing has suggested the existence of gene regulatory networks that are important for lymphoid-myeloid bifurcation ${ }^{49}$ (Figure 3). However, which component in the gene regulatory networks of these TF define lineage commitments and how the expression of the TF is altered in response to environmental changes remain unclear. The initiators of the lymphoid or myeloid lineage commitment also have yet to be clarified, and there may in fact be multiple entry points for commitment.

We previously reported that BACH factors are required for efficient commitment of multipotent progenitors and common lymphoid progenitors to the lymphoid fate..$^{17,18}$ $\mathrm{BACH}$ factors repress the expression of $\mathrm{C} / \mathrm{EBP}$, and $\mathrm{C} / \mathrm{EBP}$ repress the expression of $\mathrm{BACH}$ factors. The gene regulatory networks of these TF therefore define lymphoid or myeloid lineage commitment depending on fluctuations of the expression of $\mathrm{C} / \mathrm{EBP}$ and $\mathrm{BACH} \mathrm{TF}$ (Figure 4). Since the expression of these TF is affected by environmental changes, ${ }^{18,19,59}$ these TF may be initiators of lymphoid or myeloid lineage commitment responding to environmental changes. The development of fetal myeloid-lymphoid progenitors mentioned above ${ }^{67}$ may reflect changes in the interplay between $\mathrm{BACH}$ factors and C/EBP. Since steady-state hematopoiesis and emer-

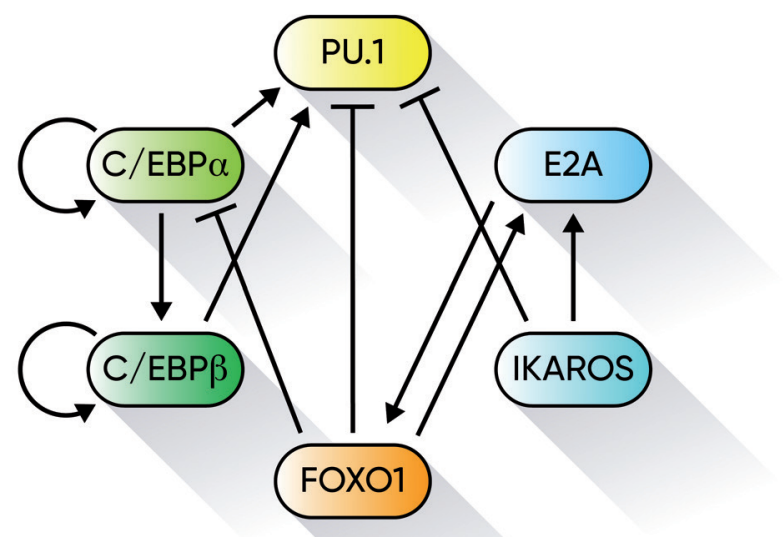

Figure 3. Gene regulatory networks controlling lymphoid cell differentiation. Several factors have been identified as important regulators of lymphoid cell differentiation commitment. ${ }^{49}$ Each factor works as an activator and/or repressor of other factors forming complex gene regulatory networks, suggesting the existence of a precise mechanism underlying lymphoid cell differentiation. However, how the activities of these factors are controlled at the initial point of lineage commitment remains unclear. gency hematopoiesis are contrasting, it is still unclear to what extent the altered expression in response to extracellular signals would affect lineage commitment. Further understanding of these issues will help to clarify the mechanisms of lineage commitment in steady-state and emergency conditions. To this end, taking advantage of using TF reporter mice exposed or not to stress might be helpful to expand the TF-based analysis further. Identification of surrogate marker genes whose expression reports activity of particular TF will also be important.

\section{Myeloid cells as the default and evolutionary prototype pathway of hematopoietic stem and progenitor cells}

The lineage commitment of HSPC is the process in which these cells lose their multipotency. For erythroid cell differentiation, the progenitor cells first lose their capacity for lymphoid differentiation, resulting in erythroid-myeloid common progenitors, ${ }^{32,35}$ at which point the decision of erythroid or myeloid lineage commitment is made. In contrast, for lymphoid cell differentiation, the progenitor cells first lose their capacity for erythroid differentiation, resulting in lymphoid-myeloid common progenitors, ${ }^{34,35}$ at which point the decision of lymphoid or myeloid lineage commitment is made.

Interestingly, it has been reported that, even after lymphoid commitment, $\mathrm{T}$-cell or B-cell progenitors retain the capacity to differentiate into myeloid cells. ${ }^{68-70}$ Indeed, myeloid differentiation potential might remain until just before terminal differentiation. Results from studies using five blood-lineage marking, which is a precise method of detecting erythroid cells and platelets in addition to myeloid, B and T cells after transplantation, also support the notion that myeloid differentiation potential is retained after losing either erythroid or lymphoid differentiation potential. ${ }^{71}$ In addition, at least some platelets are derived from HSC possessing myeloid lineage potential. ${ }^{72,73}$ Myeloid cell differentiation might, therefore, be a default and/or prototypical pathway of

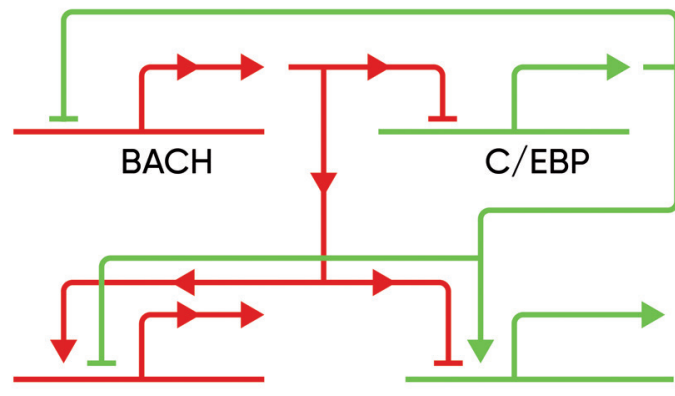

Lymphoid or erythroid genes

Myeloid genes

Figure 4. Gene regulatory networks of $\mathrm{BACH}$ and $\mathrm{C} / \mathrm{EBP}$ transcription factors for myeloid and non-myeloid gene expression. The transcription factor (TF) $\mathrm{BACH}$ represses $\mathrm{C} / \mathrm{EBP}$ and myeloid genes and induces lymphoid/erythroid genes. In contrast, the TF C/EBP represses $\mathrm{BACH}$ and lymphoid/erythroid genes and induces myeloid genes. Therefore, both stochastic fluctuation and environment-derived changes in the expression of $\mathrm{BACH}$ and $\mathrm{C} / \mathrm{EBP}$ can induce differentiation commitment in progenitor cells. 
HSPC, originally described as the "myeloid-based model" ${ }^{74}$ and repression of the myeloid differentiation program appears important for lineage commitment in HSPC. In line with this, recent reports suggest the importance of myeloid-biased HSC in emergency myelopoiesis. ${ }^{75-77}$ Further analysis is still needed to clarify how the gene regulatory networks are altered in myeloid-biased HSC.

In addition to the repression of the myeloid program during progenitor cell differentiation, re-activation of the myeloid program is observed in some mature hematopoietic cells. During maturation of erythroid and lymphoid cells, the function and expression of $\mathrm{BACH}$ factors are repressed, which can induce part of the myeloid program. For instance, Prdm1 (encoding TF BLIMP-1) is a repressed target of $\mathrm{BACH} 2$, and its repression is necessary for the proper development of $B$ cells. ${ }^{26}$ BLIMP-1 per se is necessary for the proper development of plasma cells and T cells. ${ }^{78}$ Since BLIMP-1 is important for myeloid cell development as well, ${ }^{79}$ BLIMP-1 can be considered as a part of the myeloid program deployed during plasma-cell and T-cell development. To support this notion, some of the myeloid genes are expressed in plasma cells. ${ }^{17}$ From the perspective of erythropoiesis, the expression of Hmox1 (encoding heme oxygenase-1) is induced to avoid the toxic activity of free heme during erythroblast maturation, ${ }^{80}$ On the other hand, heme oxygenase-1 is also important for the proper function of myeloid cells. ${ }^{81}$ Therefore, heme oxygenase-1 can be considered as a part of the myeloid program deployed during the development of erythroid cells. Both mature myeloid cells and non-myeloid cells (erythroid and lymphoid cells) must cope with conditions of stress (such as oxidative stress during oxygen transportation or at the site of inflammation). We therefore assume that mature hematopoietic cells may reactivate a part of the myeloid program, such as heme oxygenase-1, to protect themselves from stresses, irrespective of their lineages. The myeloid program, which is temporarily repressed upon lineage commitment, is thus referred to as the "inner myeloid", ${ }^{82}$ because part of it can be re-activated in mature cells. Given these findings, we propose "an extended myeloid-based model" of hematopoiesis, which posits that the myeloid program possesses important roles not only in hematopoietic cell differentiation but also in mature cell function.

The extended myeloid-based model with the "inner myeloid" is well understandable when the history of biological evolution is considered. Lower organisms, such as insects, possess phagocytic cells but lack erythroid and lymphoid cells. ${ }^{83}$ A human-like HSC system was recently found in the chordate Botryllus schlosseri, with stem cells generating solely cells of myeloid lineage, such as phagocytic cells and granulocytes. ${ }^{84}$ It should be noted that a BACH-like TF is present in chordates and vertebrates ${ }^{85}$ but not in lower organisms. The prototype BACH TF may restrict myeloid differentiation of HSC. Since erythroid cells and lymphoid cells arose in the hematopoietic system during the evolution of higher organisms, repressing the myeloid program in progenitor cells ("inner myeloid") might be necessary to make nonmyeloid cells (erythroid and lymphoid cells). The findings regarding the function of $\mathrm{BACH}$ factors as repressors of the "inner myeloid" may constitute the molecular foundation of the myeloid-based model of hematopoiesis and lineage commitment. The hematopoietic system in higher eukaryotes is therefore evidence of our ancient history, just like our other body systems. ${ }^{86}$

\section{Fortifying roles of BACH factors in blood homeostasis}

$\mathrm{BACH}$ factors play not only repressive roles in the myeloid program in progenitor cells but also several indispensable roles in the operation of the hematopoietic system. For instance, BACH1 works as a balancer of globins and heme during erythroid cell maturation. ${ }^{22}$ $\mathrm{BACH} 2$ is required for the development of non-IgM type plasma cells, memory B cells, regulatory $\mathrm{T}$ cells and memory $\mathrm{T}$ cells..$^{23-30,87,88}$ and therefore works as a regulator of lymphocyte effector versus non-effector differentiation. Remarkably, these functions of $\mathrm{BACH} 2$ in lymphoid cells might be explained by its binding to the AP1 site as a transcription repressor, which is in contrast to the other TF (Fos, Jun, etc.) targeting the AP-1 site, many of which work as transcription activators. ${ }^{89}$

These functions of BACH factors in erythroid and lymphoid cells can be interpreted as indicative of their role as 'fortifying factors', since they shape steady-state hematopoiesis to prepare for infection at multiple points, as described below. With regard to erythropoiesis, the hemoglobin concentration in human blood is kept around $14 \mathrm{~g} / \mathrm{dL}$ in the steady state whereas, in general, a hemoglobin concentration $<7 \mathrm{~g} / \mathrm{dl}$ is life-threatening. There is therefore sufficient capacity for erythropoiesis to endure emergency conditions. For instance, progenitor cell differentiation can be shifted toward myelopoiesis, thus promoting the innate immune defense at the expense of erythropoiesis during a state of infection. This means that BACH factors support erythropoiesis by suppressing myelopoiesis in the steady state, fortifying the system for infection. With regard to the B-cell response, IgM-secreting plasma cells work as the first line of defense against pathogens, whereas non-IgMtype plasma cells and memory B cells are produced at a later phase or after the infection as a more effective second-line defense. ${ }^{90}$ With regard to the T-cell response, effector $T$ cells provide the first line of defense against pathogens whereas regulatory $T$ cells and memory $T$ cells work to repress an excess immune response and/or to return the state to the steady condition in preparation for the next infection. ${ }^{91}$ These responses may be coordinated by the expression of BACH factors. When their expression is reduced in response to infection, IgMsecreting plasma cells and effector $\mathrm{T}$ cells are preferentially generated. Conversely, resumption of the expression of $\mathrm{BACH}$ factors leads to the generation of non-IgM plasma cells, memory B cells, regulatory $\mathrm{T}$ cells and memory $\mathrm{T}$ cells. Therefore, $\mathrm{BACH}$ factors are required to fortify the hematopoietic system as a whole, in preparation for future infections (Figure 5).

\section{Hematologic disorders as failures of BACH gene regulatory networks}

The gene regulatory networks of HSPC may explain why pathological alterations in one lineage often accom- 
Steady state

\begin{tabular}{|c|c|c|}
\hline $\begin{array}{c}\text { Erythropoiesis } \\
\text { Memory B cells } \\
\text { non-IgM plasma cells } \\
\text { regulatory T cells } \\
\text { memory T cells }\end{array}$ & $\leftarrow \mathrm{BACH}-1$ Infectious & IgM plasma cells \\
& & effector T cells \\
\hline
\end{tabular}

Figure 5. The fortifying role of $\mathrm{BACH}$ for the next emergency. $\mathrm{BACH}$ factors support erythropoiesis and repress myelopoiesis in steady-state conditions. BACH2 in particular supports the development of memory B cells, non-IgM plasma cells, regulatory $T$ cells and memory $T$ cells in the steady state while repressing the development of IgM plasma cells and effector T cells. Since BACH factors sup port the development of the cells needed for the suppression of a previous emergency reaction and the preparation for the next emergency, $\mathrm{BACH}$ factors can be considered "fortifying factors".

pany changes in other lineages in the opposite direction. Infection and inflammation cause anemia of inflammation, which is frequently observed in chronic infections and autoimmune diseases. ${ }^{8}$ Inflammatory cytokines, such as interleukin-6, induce the expression of hepcidin, resulting in the inhibition of ferroportin. ${ }^{22}$ This regulatory axis is the mechanism by which the iron supply for erythroblast maturation is limited, resulting in anemia. ${ }^{8}$ However, this mechanism may not explain how the differentiation trajectory is modulated at the erythroidmyeloid bifurcation point during an infection and in inflammatory conditions. ${ }^{6}$ In those circumstances, the expression of BACH factors is repressed in HSPC, ${ }^{19,93}$ leading to increased myelopoiesis at the expense of erythropoiesis. Therefore, the reduced activity of BACH factors might be a novel mechanism underlying anemia of inflammation.

MDS is a major hematopoietic malignancy and is caused by a clonal disorder in HSC. ${ }^{94}$ The phenotypic features of MDS, such as anemia, autoimmune reactions and transformation into acute myeloid leukemia, may also be attributed to alterations in the state of gene regulatory networks. The expression of $B A C H 2$ is repressed in MDS. ${ }^{19}$ Since the loss of BACH2 is expected to induce anemia, an inflammatory reaction and myeloid skewing of progenitors, the repression of $B A C H 2$ that has been observed in MDS patients might be one of the causes of the characteristic symptoms of MDS. A recent genomewide analysis showed that MDS clones frequently have mutations in epigenetic modifiers and splicing factors, ${ }^{95}$ suggesting that such genetic alterations may lead to a reduction in $B A C H 2$ expression. $B A C H 2$ repression is also observed in lymphocytes on aging, with PRDM1 induction in humans. ${ }^{96}$ Since the loss of BACH2 causes autoimmune-like disorders, ${ }^{28} \mathrm{BACH} 2$ repression (and the induction of the "inner myeloid") during aging may be one of the causes of aging-related inflammation. Interestingly, HSC in mice become restricted to a myeloid fate upon aging. ${ }^{97}$ This may be due to a reduction in BACH2 expression. Moreover, mutations of epigenetic modifiers, such as those observed in clonal hematopoiesis with aging ${ }^{98}$ may cause dysregulation of the repression of the "inner myeloid", resulting in myeloid skewing and inflammation. If this is the case, aging-related dysregulation of the "inner myeloid" is part of a vicious circle since inflammation per se can cause DNA mutations. ${ }^{99} B A C H 2$ haploinsufficiency in humans has been reported to cause BACH2-related immunodeficiency and autoimmunity (BRIDA), ${ }^{100}$ a finding that may further support these possibilities. In contrast, BACH2 overexpression in progenitor cells induces erythropoiesis by repressing myelopoiesis. ${ }^{19}$ Therefore, BACH factors might be new therapeutic targets of refractory anemia induced by inflammation and MDS. Further investigations will be needed in order to determine whether or not BACH2 re-activation (or "inner myeloid" repression) in aged HSC can rescue the phenotype related to aging.

\section{Conclusions}

In this review, we have highlighted recent findings concerning the differentiation of HSPC and their limitations. Novel findings from single-cell analyses suggest the need to reconsider the canonical hierarchical differentiation model of the hematopoietic system. However, we should also consider the limitations associated with these single-cell analyses, as discussed above. The myeloid-based model involving the gene regulatory networks of BACH factors may provide a further molecular basis for understanding lineage commitment, evolutionary perspectives and pathological processes of the hematopoietic system. Understanding the roles of $\mathrm{BACH}$ factors as repressors of the "inner myeloid"' and "fortifying factors" in preparation for future emergency situations will help us to develop a more comprehensive model of the hematopoietic system.

\section{Acknowledgments}

We thank Prof. Hideo Harigae, Dr. Akihiko Muto, Dr. Kyoko Ochiai and Dr. Yusho Ishii for discussion and comments on the manuscript. Studies at Tohoku University are supported by Grants-in-Aid from the Japan Society for the Promotion of Science (18H04021, 18H05374, and $15 H 02506$ to KI and 17 H06527 to HK).

\section{References}

1. Haas S, Trumpp A, Milsom MD. Causes and consequences of hematopoietic stem cell heterogeneity. Cell Stem Cell. 2018;22 (5):627-638.

2. Bianconi E, Piovesan A, Facchin F, et al. An estimation of the number of cells in the human body. Ann Hum Biol. 2013;40(6): 463-471.
3. Muckenthaler MU, Rivella S, Hentze MW, Galy B. A red carpet for iron metabolism. Cell. 2017;168(3):344-361.

4. Summers C, Rankin SM, Condliffe AM Singh N, Peters AM, Chilvers ER Neutrophil kinetics in health and disease. Trends Immunol. 2010;31(8):318-324.

5. Busch K, Klapproth K, Barile M, et al. Fundamental properties of unperturbed haematopoiesis from stem cells in vivo.
Nature. 2015;518(7540):542-546

6. Glatman Zaretsky A, Engiles JB, Hunter CA Infection-induced changes in hematopoiesis. J Immunol. 2014;192(1):27-33.

7. Doherty CP. Host-pathogen interactions: the role of iron. J Nutr. 2007;137(5):13411344

8. Weiss G, Goodnough LT. Anemia of chronic disease. N Engl J Med. 2005;352(10):10111023. 
9. Berliner N. Anemia in the elderly. Trans Am Clin Climatol Assoc. 2013;124:230-237.

10. Nikolich-Zugich J. The twilight of immunity: emerging concepts in aging of the immune system. Nat Immunol. 2018;19 (1):10-19.

11. Wang J, Geiger H, Rudolph KL. Immunoaging induced by hematopoietic stem cell aging. Curr Opin Immunol. 2011;23(4):532-536.

12. Chung SS, Park CY. Aging, hematopoiesis, and the myelodysplastic syndromes. Blood Adv. 2017;1(26):2572-2578.

13. Jose SS, Bendickova K, Kepak T, Krenova Z, Fric J. Chronic inflammation in immune aging: role of pattern recognition receptor crosstalk with the telomere complex? Front Immunol. 2017;8:1078.

14. Karamitros D, Stoilova B, Aboukhalil Z, et al. Single-cell analysis reveals the continuum of human lympho-myeloid progenitor cells. Nat Immunol. 2018;19(1):85-97.

15. Perie L, Duffy KR, Kok L, de Boer RJ, Schumacher TN. The branching point in erythro-myeloid differentiation. Cell. 2015; 163(7):1655-1662.

16. Paul F, Arkin Y, Giladi A, et al. Transcriptional heterogeneity and lineage commitment in myeloid progenitors. Cell. 2015;163(7):1663-1677

17. Itoh-Nakadai $A$, Hikota $R$, Muto $A$, et al. The transcription repressors Bach2 and Bach1 promote $B$ cell development by repressing the myeloid program. Nat Immunol 2014;15(12):1171-1180.

18. Itoh-Nakadai $A$, Matsumoto $M$, Kato $H$, et al. A Bach2-Cebp gene regulatory network for the commitment of multipotent hematopoietic progenitors. Cell Rep. 2017; 18(10):2401-2414

19. Kato H, Itoh-Nakadai A, Matsumoto M, et al. Infection perturbs Bach2- and Bach1dependent erythroid lineage 'choice' to cause anemia. Nat Immunol. 2018;19(10): 1059-1070.

20. Igarashi K, Watanabe-Matsui M. Wearing red for signaling: the heme-bach axis in heme metabolism, oxidative stress response and iron immunology. Tohoku J Exp Med. 2014;232(4):229-253.

21. Foletta VC, Segal DH, Cohen DR. Transcriptional regulation in the immune system: all roads lead to AP-1. J Leukoc Biol. 1998;63(2):139-152.

22. Kobayashi M, Kato H, Hada $\mathrm{H}$, et al. Ironheme-Bach1 axis is involved in erythroblast adaptation to iron deficiency. Haematologica. 2017:102(3):454-465.

23. Igarashi K, Kurosaki T, Roychoudhuri R. BACH transcription factors in innate and adaptive immunity. Nat Rev Immunol. 2017;17(7):437-450.

24. Kim EH, Gasper DJ, Lee SH, Plisch EH, Svaren J, Suresh M. Bach2 regulates homeostasis of Foxp3+ regulatory T cells and protects against fatal lung disease in mice. $J$ Immunol. 2014;192(3):985-995

25. Kometani K, Nakagawa R, Shinnakasu R, et al. Repression of the transcription factor Bach2 contributes to predisposition of IgG1 memory B cells toward plasma cell differentiation. Immunity. 2013;39(1):136-147.

26. Muto A, Ochiai K, Kimura Y, et al. Bach2 represses plasma cell gene regulatory network in B cells to promote antibody class switch. EMBO J. 2010;29(23):4048-4061.

27. Muto A, Tashiro S, Nakajima O, et al. The transcriptional programme of antibody class switching involves the repressor Bach2. Nature. 2004;429(6991):566-571.

28. Roychoudhuri R, Hirahara K, Mousavi K, et al. $\mathrm{BACH} 2$ represses effector programs to stabilize T(reg)-mediated immune homeostasis. Nature. 2013;498(7455):506-510.

29. Tsukumo S, Unno M, Muto A, et al. Bach2 maintains $T$ cells in a naive state by suppressing effector memory-related genes. Proc Natl Acad Sci U S A. 2013;110(26): 10735-10740

30. Yu X, Lao Y, Teng XL, et al. SENP3 maintains the stability and function of regulatory $\mathrm{T}$ cells via BACH2 deSUMOylation. Nature Commun. 2018;9(1):3157.

31. Yamamoto R, Morita Y, Ooehara J, et al. Clonal analysis unveils self-renewing lineage-restricted progenitors generated directly from hematopoietic stem cells. Cell. 2013;154(5):1112-1126.

32. Akashi K, Traver D, Miyamoto T, Weissman IL. A clonogenic common myeloid progenitor that gives rise to all myeloid lineages. Nature. 2000;404(6774):193-197.

33. Boyer SW, Schroeder AV, Smith-Berdan S, Forsberg EC. All hematopoietic cells develop from hematopoietic stem cells through Flk2/Flt3-positive progenitor cells. Cell Stem Cell. 2011:9(1):64-73.

34. Adolfsson J, Mansson R, Buza-Vidas N, et al. Identification of Flt3+ lympho-myeloid stem cells lacking erythro-megakaryocytic potential a revised road map for adult blood lineage commitment. Cell. 2005;121(2):295-306.

35. Pietras EM, Reynaud D, Kang YA, et al Functionally distinct subsets of lineagebiased multipotent progenitors control blood production in normal and regenerative conditions. Cell Stem Cell. 2015;17 (1):35-46.

36. Naik SH, Perie L, Swart E, et al. Diverse and heritable lineage imprinting of early haematopoietic progenitors. Nature. 2013:496(7444):229-232.

37. Yu VWC, Yusuf RZ, Oki T, et al. Epigenetic memory underlies cell-autonomous heterogeneous behavior of hematopoietic stem cells. Cell. 2016;167(5):1310-1322.e1317.

38. Imayoshi I, Isomura A, Harima Y, et al. Oscillatory control of factors determining multipotency and fate in mouse neural progenitors. Science. 2013;342(6163):12031208.

39. Morrison SJ, Scadden DT. The bone marrow niche for haematopoietic stem cells. Nature. 2014:505(7483):327-334.

40. Busch K, Rodewald HR. Unperturbed vs. post-transplantation hematopoiesis: both in vivo but different. Curr Opin Hematol. 2016;23(4):295-303

41. Nimmo RA, May GE, Enver T. Primed and ready: understanding lineage commitment through single cell analysis. Trends Cell Biol. 2015;25(8):459-467.

42. Skylaki S, Hilsenbeck O, Schroeder T. Challenges in long-term imaging and quantification of single-cell dynamics. Nat Biotechnol. 2016;34(11):1137-1144

43. Golan K, Kumari A, Kollet O, et al. Daily onset of light and darkness differentially controls hematopoietic stem cell differentiation and maintenance. Cell Stem Cell. 2018;23(4):572-585.e577.

44. Teschendorff AE, Enver T. Single-cell entropy for accurate estimation of differentiation potency from a cell's transcriptome Nat Commun. 2017:8:15599.

45. Wang X, Allen WE, Wright MA, et al. Threedimensional intact-tissue sequencing of single-cell transcriptional states. Science. 2018;361(6400)

46. Rodriguez-Fraticelli AE, Wolock SL, Weinreb CS, et al. Clonal analysis of lineage fate in native haematopoiesis. Nature. 2018;553(7687):212-216.

47. Pei W, Feyerabend TB, Rossler J, et al.
Polylox barcoding reveals haematopoietic stem cell fates realized in vivo. Nature. 2017;548(7668):456-460.

48. Wolff L, Humeniuk R. Concise review: erythroid versus myeloid lineage commitment: regulating the master regulators. Stem Cells 2013;31(7):1237-1244.

49. Collombet S, van Oevelen C, Sardina Ortega JL, et al. Logical modeling of lymphoid and myeloid cell specification and transdifferentiation. Proc Natl Acad Sci U S A. 2017;114(23):5792-5799.

50. Teles I, Pina C, Eden P, Ohlsson M, Enver T, Peterson C. Transcriptional regulation of lineage commitment--a stochastic model of cell fate decisions. PLoS Comput Biol. 2013;9(8):e1003197.

51. Liston A, Masters SL. Homeostasis-altering molecular processes as mechanisms of inflammasome activation. Nat Rev Immunol. 2017;17(3):208-214.

52. Laurenti E, Gottgens B. From haematopoietic stem cells to complex differentiation landscapes. Nature. 2018;553(7689):418-426.

53. Heyworth C, Pearson S, May G, Enver T. Transcription factor-mediated lineage switching reveals plasticity in primary committed progenitor cells. EMBO J. 2002;21 (14):3770-3781

54. Pina C, Teles J, Fugazza C, et al. Single-cell network analysis identifies DDIT3 as a nodal lineage regulator in hematopoiesis. Cell Rep. 2015;11(10):1503-1510.

55. Mayran A, Drouin J. Pioneer transcription factors shape the epigenetic landscape. J Biol Chem. 2018;293(36):13795-13804

56. Bonifer C. Epigenetic plasticity of hematopoietic cells. Cell Cycle. 2005;4(2): 211-214.

57. Herb BR, Wolschin F, Hansen KD, et al Reversible switching between epigenetic states in honeybee behavioral subcastes. Nat Neurosci. 2012:15(10):1371-1373.

58. Avellino R, Delwel R. Expression and regulation of C/EBPalpha in normal myelopoiesis and in malignant transformation. Blood. 2017;129(15):2083-2091.

59. Hirai H, Zhang P, Dayaram T, et al $\mathrm{C} / \mathrm{EBPbeta}$ is required for 'emergency' granulopoiesis. Nat Immunol. 2006;7(7):732-739.

60. Iwasaki H, Somoza C, Shigematsu H, et al. Distinctive and indispensable roles of PU.1 in maintenance of hematopoietic stem cells and their differentiation. Blood. 2005;106 (5):1590-1600

61. Fujiwara Y, Browne CP, Cunniff K, Goff SC, Orkin SH. Arrested development of embryonic red cell precursors in mouse embryos lacking transcription factor GATA-1. Proc Natl Acad Sci U S A. 1996;93(22):1235512358.

62. Hoppe PS, Schwarzfischer M, Loeffler D, et al. Early myeloid lineage choice is not initiated by random PU.1 to GATA1 protein ratios. Nature. 2016;535(7611):299-302.

63. Palii CG, Cheng Q, Gillespie MA, et al Single-cell proteomics reveal that quantitative changes in co-expressed lineage-specific transcription factors determine cell fate. Cell Stem Cell. 2019;24(5):812-820.e815.

64. Weinberg ED. Iron loading and disease surveillance. Emerg Infect Dis. 1999;5(3):346352.

65. Yanez A, Goodridge HS, Gozalbo D, Gil ML. TLRs control hematopoiesis during infection. Eur J Immunol. 2013;43(10):25262533

66. Hsu W, Kerppola TK, Chen PL, Curran T, Chen-Kiang S. Fos and Jun repress transcription activation by NF-IL6 through association at the basic zipper region. Mol Cell Biol. 1994:14(1):268-276. 
67. Boiers C, Richardson SE Laycock E, et al. A human IPS model implicates embryonic Bmyeloid fate restriction as developmental susceptibility to B acute lymphoblastic leukemia-associated ETV6-RUNX1. Dev Cell. 2018:44(3):362-377.e367.

68. Kawamoto H, Ohmura K, Katsura Y. Presence of progenitors restricted to T, B, or myeloid lineage, but absence of multipotent stem cells, in the murine fetal thymus. J Immunol. 1998;161(8):3799-3802.

69. Wada H, Masuda K, Satoh R, et al. Adult Tcell progenitors retain myeloid potential. Nature. 2008;452(7188):768-772.

70. Kawamoto H, Ohmura K, Katsura Y. Direct evidence for the commitment of hematopoietic stem cells to T, B and myeloid lineages in murine fetal liver. Int Immunol. 1997;9(7):1011-1019.

71. Yamamoto R, Wilkinson AC, Nakauchi H. Changing concepts in hematopoietic stem cells. Science. 2018;362(6417):895-896.

72. Sanjuan-Pla A, Macaulay IC, Jensen CT, et al. Platelet-biased stem cells reside at the apex of the haematopoietic stem-cell hierarchy. Nature. 2013;502(7470):232-236.

73. Carrelha J, Meng Y, Kettyle LM, et al. Hierarchically related lineage-restricted fates of multipotent haematopoietic stem cells. Nature. 2018;554(7690):106-111.

74. Kawamoto H. A close developmental relationship between the lymphoid and myeloid lineages. Trends Immunol. 2006;27(4):169-175.

75. Pietras EM. Inflammation: a key regulator of hematopoietic stem cell fate in health and disease. Blood. 2017;130(15):1693-1698.

76. Mann M, Mehta A, de Boer CG, et al. Heterogeneous responses of hematopoietic stem cells to inflammatory stimuli are altered with age. Cell Rep. 2018;25(11): 2992-3005.e2995.

77. Dutta P, Sager HB, Stengel KR, et al. Myocardial infarction activates CCR2(+) hematopoietic stem and progenitor cells. Cell Stem Cell. 2015;16(5):477-487.

78. Fu SH, Yeh LT, Chu CC, Yen BL, Sytwu HK. New insights into Blimp-1 in T lymphocytes: a divergent regulator of cell destiny and effector function. I Biomed Sci. 2017;24(1):49.

79. Chang DH, Angelin-Duclos C, Calame K. BLIMP-1: trigger for differentiation of myeloid lineage. Nat Immunol. 2000;1(2): 169-176.

80. Garcia-Santos D, Schranzhofer M Horvathova M, et al. Heme oxygenase 1 is expressed in murine erythroid cells where it controls the level of regulatory heme. Blood. 2014;123(14):2269-2277.

81. Tzima S, Victoratos P, Kranidioti K, Alexiou M, Kollias G. Myeloid heme oxygenase-1 regulates innate immunity and autoimmunity by modulating IFN-beta production. J Exp Med. 2009;206(5):1167-1179.

82. Igarashi K, Itoh-Nakadai A. Orchestration of B lymphoid cells and their inner myeloid by Bach. Curr Opin Immunol. 2016;39(136142.

83. Krzemien J, Crozatier M, Vincent A. Ontogeny of the Drosophila larval hematopoietic organ, hemocyte homeostasis and the dedicated cellular immune response to parasitism. Int J Dev Biol 2010;54(6-7):1117-1125

84. Rosental B, Kowarsky M, Seita J, et al Complex mammalian-like haematopoietic system found in a colonial chordate. Nature. 2018;564(7736):425-429.

85. Amoutzias GD, Veron AS, Weiner J, 3rd, et al. One billion years of bZIP transcription factor evolution: conservation and change in dimerization and DNA-binding site specificity. Mol Biol Evol. 2007;24(3):827-835.

86. Shubin N. Your Inner Fish: A Journey into the 3.5-Billion-Year History of the Human Body. Knopf Doubleday Publishing Group. 2008.

87. Shinnakasu R, Inoue $\mathrm{T}$, Kometani $\mathrm{K}$, et al. Regulated selection of germinal-center cells into the memory B cell compartment. Nat Immunol. 2016;17(7):861-869.

88. Roychoudhuri R, Clever D, Li P, et al. $\mathrm{BACH} 2$ regulates $\mathrm{CD} 8(+) \mathrm{T}$ cell differentiation by controlling access of AP-1 factors to enhancers. Nat Immunol. 2016;17(7):851860.

89. Sidwell $\mathrm{T}$, Kallies A. Bach2 is required for $\mathrm{B}$ cell and T cell memory differentiation. Nat Immunol. 2016;17(7):744-745.

90. Baumgarth N. B-1 cell heterogeneity and the regulation of natural and antigen-induced IgM production. Front Immunol. 2016 $7(324$.

91. Hall BM. T Cells: soldiers and spies--the surveillance and control of effector $T$ cells by regulatory T cells. Clin J Am Soc Nephrol. 2015:10(11):2050-2064.

92. Abreu R, Quinn F, Giri PK. Role of the hepcidin-ferroportin axis in pathogen-mediated intracellular iron sequestration in human phagocytic cells. Blood Adv. 2018;2(10): 1089-1100.

93. Mitroulis I, Ruppova K, Wang B, et al Modulation of myelopoiesis progenitors is an integral component of trained immunity. Cell. 2018:172(1-2):147-161.e112.

94. Tefferi A, Vardiman JW. Myelodysplastic syndromes. N Engl J Med. 2009;361(19): 1872-1885.

95. Yoshida K, Sanada M, Shiraishi Y, et al Frequent pathway mutations of splicing machinery in myelodysplasia. Nature. 2011;478(7367):64-69

96. Chi VLD, Garaud S, De Silva P, et al. Agerelated changes in the BACH2 and PRDM genes in lymphocytes from healthy donor and chronic lymphocytic leukemia patients. BMC Cancer. 2019;19(1):81.

97. Yamamoto R, Wilkinson AC, Ooehara J, et al. Large-scale clonal analysis resolves aging of the mouse hematopoietic stem cell compartment. Cell Stem Cell. 2018;22(4):600607.e604.

98. Loh PR, Genovese G, Handsaker RE, et al. Insights into clonal haematopoiesis from 8,342 mosaic chromosomal alterations. Nature. 2018;559(7714):350-355

99. Kawanishi S, Ohnishi S, Ma N, Hiraku Y Murata M. Crosstalk between DNA damage and inflammation in the multiple steps of carcinogenesis. Int J Mol Sci. 2017;18(8).

100. Afzali B, Gronholm J, Vandrovcova J, et al. BACH2 immunodeficiency illustrates an association between super-enhancers and haploinsufficiency. Nat Immunol. 2017;18 (7):813-823. 